\title{
Configuração do processo ofensivo no jogo de Andebol pela rela- ção cooperação/oposição relativa à zona da bola. Estudo em equi- pas portuguesas de diferentes níveis competitivos
}

\author{
Ireneu Moreira \\ Fernando Tavares
}

https://doi.org/10.5628/rpcd.04.01.29

\section{RESUMO}

Este estudo teve como objectivo analisar e comparar a configuração do processo ofensivo no jogo de andebol, em equipas masculinas portuguesas de distintos níveis de competição, considerando a relação cooperação/oposição relativa à zona da bola. A amostra é composta por dois grupos distintos: (i) o Grupo Sénior (GS), constituído pelas quatro equipas melhores classificadas no Campeonato Nacional da I ${ }^{\mathrm{a}}$ divisão, com a observação de dez jogos; (ii) o Grupo Juvenil (GJ), constituído pelas cinco equipas que disputaram a final do Campeonato

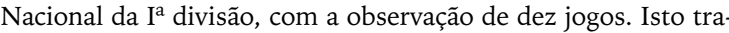
duz-se num total de 1365 sequências ofensivas, 980 para o GS e 385 para GJ. O método utilizado foi o de observação indirecta, tendo como técnica a observação sistemática de imagens gravadas. No sentido de proceder à observação do processo ofensivo tivemos em consideração: (i) sequência de passes na construção do processo ofensivo anterior à perda da posse da bola; (ii) condições de finalização; e (iii) acção de finalização. Para a anotação dos dados foi elaborada uma ficha de observação. A análise dos dados foi efectuada a partir dos procedimentos da estatística descritiva. Para comparar as diferenças entre os dois grupos foi aplicado o teste one way Anova. Os resultados obtidos permitem concluir que: (i) os dois grupos utilizam os métodos de jogo de acordo com estruturas idênticas aos padrões internacionais e conforme os padrões de identidade da própria modalidade, sendo portanto o jogo em ataque posicional o mais utilizado - no GJ com $85 \%$ e no GS com $76 \%$ dos ataques; (ii) é preocupante a ausência dos métodos de jogo no grande espaço nos dois grupos, especialmente no GJ; (iii) em ambos os grupos, as condições de finalização em igualdade numérica relativa são as mais frequentes; (iv) os jogadores do GS conseguem converter estas situações de igualdade em vantagem, aquando da perda de posse da bola; (v) nas situações de assimetria, ambos os grupos apresentam, no ataque posicional, características idênticas no aumento/redução da relação numérica relativa, não sendo muito claro o aproveitamento da situação no GJ; e (vi) não se verificaram diferenças na solicitação das diferentes linhas dos sistemas de jogo, em ambos os gru-pos, sendo a preponderância da primeira linha do sistema de ataque comum.

Palavras-chave: andebol, análise do jogo, rendimento, processo ofensivo, simetria/assimetria numérica.
Faculdade de Ciências do Desporto e de Educação Física Universidade do Porto, Portugal

\section{ABSTRACT}

Configuration of offensive process in Portuguese handball male teams of different competitive levels considering the relationship co-operation/opposition at the ball area.

The purpose of this study was to analyse and compare the handball attacking process in Portuguese male teams of different competitive levels, considering the relationship co-operation/opposition at the ball area. Sample includes two different groups: (i) the Senior Group (SG), consisting of the four best National League ranked teams (ten games analysed); (ii) the Youth Group (YG), consisting of the 5 teams who participated in the final tournament of the Juvenile National League (five games analysed). In total, 1365 attacking sequences were studied (980 for SG and 385 for YG). Indirect observation based on films of all games was used. In order to analyse the attacking process we considered (i) the passing sequence used to build the attacking process previous to the loss of the ball; (ii) the finishing conditions; and (iii) the finishing action. Data has been registered in a specific observation form. Descriptive statistics and one way Anova was used for data analysis. Results support the following conclusions: (i) in both groups, game method structure is similar to international standards. Positional attack is the most used method - 85\% in YG and $76 \%$ in SG; (ii) "large space" game methods must be implemented on both groups, specially on YG; (iii) in both groups the best finishing conditions are those in relative numerical equality; (iv) SG players usually turn these situations into advantage during loss of ball possession situations; $(v)$ in absolute asymmetrical situations of positional attack, both groups show similar performance in increasing/decreasing the relative numerical relation during the final phase; and (vi) concerning the participation of the different positions of game systems, there are no differences between the groups. First line of attack prevails in both groups.

Key Words: handball, match analysis, performance, offensive process, numerical symmetry/asymmetry. 


\section{INTRODUÇÃO}

No jogo de Andebol a base do êxito passa por obter, como consequência de colocação adequada, uma vantagem numérica numa determinada parte ou zona do campo (11). Esta vantagem numérica é habitualmente designada por superioridade numérica, a qual pode ser absoluta ou relativa, independentemente do facto de se verificar no ataque ou na defesa (23).

A superioridade numérica absoluta verifica-se em resultado de disposições regulamentares e a superioridade numérica relativa quando, por questões decorrentes do próprio jogo, se cria essa vantagem numérica, através de uma gestão racional do tempo e espaço, nomeadamente nas acções que se desenvolvem no jogo $(7,11)$. A manifestação dessa vantagem numérica, a qual inclui a vantagem posicional, verifica-se nas condições favoráveis para acções de finalização ou de manutenção da posse da bola (41). O Andebol apresenta uma particularidade de identidade nas regras de jogo que é realçada no próprio jogo, pois pode prever-se situações de superioridade/inferioridade numérica garantidas à partida: (i) quando se trata de sanções que consideram a exclusão, desclassificação e expulsão (regra 16); ou (ii) particularidades regulamentares do próprio jogo, como o caso do número de jogadores exigidos para iniciar o jogo (regra 4:1) (18).

\section{A criação da superioridade numérica relativa no ataque}

A construção de situações de superioridade numérica relativa no jogo ofensivo, no Andebol, é, para vários autores, a intenção dominante, de acordo com a qual os jogadores desenvolvem as suas acções ofensivas, procurando criar situações privilegiadas de finalização $(2,4,5,11,24,25,26,27,29,36,38,40,42)$. Habitualmente designadas por princípios $(35,41)$, estas regras gerais, ou de base, devem ser consideradas como princípios fundamentais $(19,35)$ : (i) devendo-se recusar a inferioridade numérica relativa; (ii) evitar a igualdade numérica relativa; e (iii) procurar criar a superioridade numérica relativa.

\section{Métodos de jogo}

As formas de organização das acções dos jogadores obedecem a princípios fundamentais que visam a racionalização do processo ofensivo, sendo que esta forma geral de organização das acções dos jogadores no ataque - método de jogo ofensivo - manifesta-se dentro do sistema de jogo previamente definido pela equipa (6). O sistema de jogo representa o modo de colocação de base dos jogadores no terreno de jogo, sendo uma estrutura fundamental da táctica colectiva $(6,41)$.

Assim, podemos considerar: (i) contra-ataque, ou jogo no grande espaço, jogo na totalidade do terreno de jogo disponível, suportado na rápida concretização das intenções de ataque; (ii) ataque rápido, um híbrido de jogo no grande espaço e de espaço reduzido, em que a equipa que procurou promover o contra-ataque não obteve êxito e encadeou essas acções com as do ataque posicional, pressupondo, ou não, este encadeamento um momento de mudança de ritmo (36); e (iii) o ataque posicional, ou jogo no espaço reduzido, em zona próxima da baliza, junto ao limite da área de baliza e onde se verifica a maior densidade de jogo $(10,38)$.

\section{A circulação da bola e a circulação de jogadores}

O objectivo geral da circulação da bola é a mobilização e desequilíbrio do adversário, e a exploração desses desequilíbrios, sendo a qualidade da circulação da bola fundamental para a exploração desses desequilíbrios (21). Muitas vezes estas acções são complementadas pela circulação de jogadores, ou seja, quando um ou mais jogadores procuram espaços livres fora dos seus postos específicos, o que exige coordenação de acções, disciplina colectiva e organização táctica. Este é um meio muito utilizado no andebol e desenvolve-se em estreita relação com a circulação da bola (22).

$\mathrm{O}$ ataque posicional apresenta, de forma cada vez mais frequente, o sistema de jogo 3:3, como base para passagem a 2:4 $(1,28,39)$. Os sistemas de ataque mais utilizados, e quase que exclusivamente referenciados, são o 3:3 e o 2:4. Por vezes nem surgem referências directas aos sistemas de ataque ( 9 , $32,26)$, sendo que é de uma forma geral considerado que o ataque se desenvolve a partir de um sistema 3:3, e em algumas situações e no decorrer das acções de ataque anterior à finalização, com a circulação da bola e de jogadores passa a 2:4. 


\section{Meios tácticos de grupo}

A utilização de meios tácticos de grupo revela-se como o complemento dos sistemas de jogo, permitindo alguma criatividade a partir de combinações simples de dois ou três jogadores $(12,20,42)$. A táctica de grupo, como parte da táctica colectiva, compreende o trabalho coordenado das interacções directas entre dois ou mais jogadores, com o objectivo de criar possibilidades de superioridade ou aproveitar as situações de igualdade numérica. Em caso algum deve ser interpretado como uma jogada pré-fabricada, mas como meio de ataque natural e flexível (3), como são exemplos: (i) o passa-e-vai, (ii) as penetrações sucessivas (progressões sucessivas ou apoios móveis), (iii) o cruzamento, (iv) o bloqueio (bloqueio, ecran e cortina) e (v) as trocas (trocas ou trocas de posto específico).

\section{A importância do passe no jogo}

A utilização de meios tácticos colectivos não tem relação directa com a conclusão de sequências ofensivas finalizadas com ou sem remate, sendo muito claro que essa finalização é suportada por meios tácticos individuais (31). O passe é um elemento técnico de base, mas muito importante na construção das sequências ofensivas, pelo que deve ser seguro, preciso, rápido, oportuno e inteligente (38). Este autor sublinha que estas características são determinantes para garantir a posse de bola, evitando a iniciativa do adversário, a não ser após a realização de remate à baliza com possibilidade de golo.

\section{Objectivo}

No presente estudo pretendemos analisar o jogo das melhores equipas portuguesas de distintos níveis competitivos (jovens e adultos), de forma a configurar a organização ofensiva pela superioridade numérica relativa destes dois grupos. Recorremos à análise das condições de finalização, condições essas criadas pela equipa ou pelo jogador, traduzida pela relação numérica de cooperação/oposição nas condições em que se verifica a perda da posse da bola.

Pretendemos desta forma poder, para os dois níveis competitivos: (i) descrever e comparar a incidência de utilização dos distintos métodos de jogo; (ii) descrever e comparar a relação numérica de cooperação/oposição, relativa à zona da bola, nas condições de finalização e de perda da posse da bola; (iii) relacionar as situações de desigualdade absoluta, nos métodos de jogo, com as condições de finalização e perda de posse da bola; e (iv) configurar o jogo.

\section{MATERIAL E MÉTODOS \\ Amostra}

Para a realização do presente estudo foram escolhidos dois níveis competitivos, de acordo com o escalão etário, distintos do andebol masculino português: (i) equipas apuradas para o grupo A (disputa do título nacional) do Campeonato Nacional da Ia Divisão Sénior, na época de 1997/98, que constituem o grupo sénior (GS); e (ii) as equipas que disputaram o Campeonato Nacional da I ${ }^{\text {a }}$ Divisão de Juvenis, na época de 1997/98, que constituem o grupo juvenil (GJ).

A amostra foi constituída a partir de um total de quinze jogos, dez do grupo sénior (GS) e cinco do grupo juvenil (GJ), com uma composição de 1365 sequências ofensivas, 980 para o grupo do escalão sénior e de 385 para o grupo do escalão juvenil. O método de observação utilizado no presente estudo é o método de observação indirecta, tendo como técnica a observação sistemática de imagens gravadas (33).

No sentido de proceder à observação do processo ofensivo tivemos em consideração: (i) sequência de passes na construção do processo ofensivo anterior à perda da posse da bola (passes de ataque); (ii) condições de finalização; e (iii) acção de finalização.

\section{Categorização e explicitação das variáveis}

A definição das categorias e dos elementos de análise foi testada na forma de fichas de observação. Nesta foram considerados o número de ataques (NA), o número de passes de ataque (NPA) e as linhas (Lh) do sistema de jogo posicional, sendo designada por $1^{a}$ linha a que integra os jogadores que estão na zona mais próxima da nossa área de baliza e por $2^{a}$ linha a que integra os jogadores que estão na zona mais afastada (36). Foram também considerados, para efeitos de registo, os distintos métodos de jogo: (i) contra-ataque (CA); (ii) ataque rápido (AR); e (ii) ataque posicional (AP).

Diferenciou-se a superioridade numérica absoluta (SPA) e a inferioridade numérica absoluta (INA), 
considerando as regras de jogo (4:1, 16:3 e 16:9) (18), da superioridade numérica relativa (SuNR), da igualdade numérica relativa (IgNR) e da inferioridade numérica relativa (IfNR), que se podiam verificar em quaisquer situações de relação numérica absoluta. Designamos por condiçôes de finalização (CF) uma situação de cooperação/oposição que se verifica até ao momento da decisão de finalizar e registamos a relação numérica relativa à zona da bola.

Consideramos ainda como distintas as condições de perda de posse da bola (PB) (41): (i) normal (Nr), sempre que é obtido golo; (ii) justificada (Js), sempre que se tentou obter golo mas sem êxito; e (iii) não justificada $(\mathrm{Nj})$, quando por erro ou desrespeito pelo regulamento, bem como outras situações não previstas, se perde a posse da bola.

\section{Fiabilidade da observação}

As observações para determinar a fiabilidade intra-observador processaram-se com um intervalo superior a três meses. O nível de mais de $90 \%$, obtido nas diferentes medições, num sistema complexo de observação é considerado fiável (43).

\section{Procedimentos estatísticos}

A análise dos dados foi efectuada a partir dos procedimentos da estatística descritiva apresentando-se a média, o desvio-padrão, a amplitude de variação e a percentagem.

Para identificar diferenças entre os grupos considerados recorreu-se ao teste one way Anova. O nível de significância foi mantido em $5 \%$.

\section{APRESENTAÇÃO E DISCUSSÃO DOS RESULTADOS}

O número médio de ataques que cada equipa apresenta por jogo, nas provas mundiais, tem aumentado nesta última década, registando valores como 47,9 em 1993 (Campeonato do Mundo Masculino, Suécia), 52,6 em 1995 (Campeonato do Mundo Masculino, Islândia) e 54,3 em 1999 (Campeonato do Mundo Masculino, Egipto) $(34,37)$.

\section{Os métodos de jogo}

Os resultados, no Quadro 1, permitem salientar que apenas o Grupo Juvenil apresenta um menor número de ataques por jogo.
A elevada percentagem de jogo posicional, só por si, realça a importância do método de jogo em ataque posicional no jogo de Andebol e estabelece uma relação entre o predomínio do método e o ritmo de jogo. $\mathrm{O}$ método de jogo em ataque posicional, método dominante, situa-se em valores que oscilam entre os $60 \%(12,14,20,30)$ e os $80 \%(8,24,25,30,40)$, sendo que os valores obtidos no nosso estudo apresentam valores superiores a $80 \%$ (indicador de forte "colonização" pelo método de jogo em ataque posicional) para o Grupo Juvenil $(84,6 \%)$ e dentro do intervalo referido para o Grupo Sénior (76,19\%). Estes valores do Grupo Sénior são próximos aos apresentados em outros estudos $(75 \%$ e $73,2 \%)(2,31)$.

Quadro 1 - Número total de ataques relativos aos métodos de jogo (ataque posicional, contra ataque e ataque rápido], percentagem e média por jogo, relativamente aos dois grupos (GS e GJ).

\begin{tabular}{l|c|c|c|c|c|c|c|c} 
& \multicolumn{3}{|c}{ Sénior [GS] } & \multicolumn{3}{c|}{ Juvenil (GJ) } & \multicolumn{2}{c}{} \\
\hline & $\mathrm{n}$ & $\%$ & $\mathrm{~m}$ & $\mathrm{n}$ & $\%$ & $\mathrm{~m}$ & $\mathbf{F}$ & $\mathbf{p}$ \\
\hline Ataque posicional & 739 & 76,19 & 36,95 & 324 & 84,60 & 32,4 & 1,924 & 0,169 \\
\hline Contra ataque & 193 & 19,90 & 9,65 & 38 & 9,92 & 3,8 & 19,114 & $\mathbf{0 , 0 0 0}$ \\
\hline Ataque rápido & 38 & 3,92 & 1,9 & 21 & 5,48 & 2,1 & 0,660 & 0,798 \\
\hline Total & 970 & & 48,5 & 383 & & 38,3 & &
\end{tabular}

Estes resultados apresentam uma disparidade aceitável para validar a ordem de importância dos diferentes métodos de jogo. É no método de jogo no grande espaço (contra-ataque) que surgem as maiores divergências nos registos efectuados nos diferentes estudos $(2,9,12,20,30,31)$. Neste estudo, a diferença encontrada é estatisticamente significativa $(0,00)$, já que o Grupo Juvenil apresenta um recurso diminuto do método de jogo em contra-ataque $(9,92 \%)$, tanto em termos absolutos como relativamente ao Grupo Sénior $(19,90 \%)$. Os valores registados para o método de jogo em ataque rápido destacam a sua diminuta utilização em ambos os grupos (Grupo Sénior com 3,92\% e Grupo Juvenil com 2,1\%). A solicitação dos diferentes métodos de jogo traduz opções estratégicas importantes. Essas opções devem considerar as tendências modernas do jogo, que são a rapidez e o dinamismo (16) e procurar 
soluções tácticas e técnicas relevantes para tornar o jogo mais aliciante (15). Não foi exactamente isto que encontrámos nos dois grupos que estudámos, pois apresentam resultados que não se enquadram no jogo de andebol actual, de nível internacional, sendo claro que o Grupo Juvenil tem necessidade de desenvolver mais o jogo no grande espaço.

\section{As condições de finalização e a perda de posse de bola nos diferentes métodos de jogo}

As características que apresentam as equipas dos dois grupos, relativamente às condições de finalização e da perda de posse da bola nos diferentes métodos de jogo, são indicadores de relevo neste estudo, pelo que a sua análise foi efectuada com fraccionamento e agrupamento de dados, de acordo com as situações de assimetria ou simetria numérica relativa. Para cada uma das distintas situações de relação numérica (superioridade, igualdade e inferioridade numérica relativa) podemos ter diferentes situações de ocorrência de perda de posse de bola (normal, justificada e não justificada), sendo que, no momento desta, se podem encontrar diferentes formas de relação numérica. Foram ainda consideradas nesta análise todas as situações de simetria absoluta, igualdade numérica absoluta, verificadas durante os jogos e analisadas relativamente aos diferentes métodos de jogo.

Ataque posicional em simetria absoluta Este método de jogo apresenta no Andebol, em particular em igualdade numérica absoluta (simetria), grande importância, pela frequência. Verificamos que, relativamente ao ataque posicional em simetria, os valores apresentados (quadro 2) para as condições de finalização não registaram qualquer diferença estatisticamente significativa.

Quadro 2 - Valores das condições de finalização que a equipa criou em ataque posicional, com média ( $\mathrm{m}$ ) desvio-padrão (DP), amplitude, para cada um dos grupos, e os valores de $F$ e $p$ referentes à comparação dos grupos.

\begin{tabular}{|c|c|c|c|c|c|c|c|c|c|}
\hline \multicolumn{2}{|c|}{ Condições de finalização } & \multicolumn{3}{|c|}{ Seniores } & \multicolumn{3}{|c|}{ Juvenis } & & \multirow[b]{2}{*}{$\mathrm{p}$} \\
\hline Ataque Posicional & & $\mathrm{m}$ & $+\mathrm{DP}$ & amplitude & $\mathrm{m}$ & $+\mathrm{DP}$ & amplitude & & \\
\hline & Superioridade numérica relativa & 8,95 & $\pm 3,44$ & $3-14$ & 9,90 & $\pm 2,73$ & $5-14$ & $0,5>7$ & 0,454 \\
\hline & |gualdade numérica relativa & 15,25 & +4.38 & $8-23$ & 13,30 & $+2,41$ & $10-19$ & 1,707 & 0,202 \\
\hline & Inferioridade numérica relativa & 6,45 & $\pm 2,61$ & $3 \cdot 13$ & 5,10 & $\pm 2,28$ & $2 \cdot 9$ & 1,935 & 0,175 \\
\hline
\end{tabular}

O mesmo se verifica no que se refere à perda de posse de bola (Quadro 3), uma vez que devemos considerar que o valor encontrado na comparação dos dois grupos, apenas em superioridade numérica relativa apresenta um valor de pouco significado estatístico $(0,092)$. Admitimos que a exigência de análise poderá permitir, consoante o caso do estudo em causa, que o valor tenha significado ou não (17), mas enten- demos como adequado, no caso do presente estudo, que não tenha significado estatístico, podendo no entanto ser considerado como um indicador de análise. Nesta perspectiva, podemos sublinhar que a competência individual, no Grupo Sénior, permite valorizar de forma acentuada a superioridade numérica relativa no momento de perda de posse de bola, relativamente às condições de finalização criadas.

Quadro 3 - Valores da perda da posse de bola que a equipa criou em ataque posicional, com média (m) desvio-padrão (DP), amplitude, para cada um dos grupos, e os valores de $F$ e $p$ referentes à comparação dos grupos.

\begin{tabular}{|c|c|c|c|c|c|c|c|c|c|}
\hline \multicolumn{2}{|c|}{ Perda da posse de bola } & \multicolumn{3}{|c|}{ Seniores } & \multicolumn{3}{|c|}{ Juvenis } & \multirow[b]{2}{*}{$\mathrm{F}$} & \multirow[b]{2}{*}{$p$} \\
\hline Ataque Posicional & & $\mathrm{m}$ & $\pm D P$ & amplitude & $\mathrm{m}$ & $\pm \mathrm{DP}$ & amplitude & & \\
\hline & Superioridade numérica relativa & 13.35 & $+3,51$ & $8-21$ & 11,10 & $+2,88$ & 7.18 & 3,054 & 0,092 \\
\hline & Igualdade numérica relativa & 11,50 & $+4,38$ & $6-19$ & 11,80 & $+2,15$ & $9-17$ & 0,041 & 0,840 \\
\hline & Inferioridade numérica relativa & 5,75 & $+2,65$ & $1-11$ & 5,10 & $+2,64$ & $1-10$ & 0,401 & 0,532 \\
\hline
\end{tabular}


Ambos os grupos têm dificuldade em operacionalizar a intenção táctica - superioridade numérica relativa como condição de finalização, mas na forma como se verifica a perda de posse de bola esta apresenta-se como dominante para o Grupo Sénior. Para tornar mais claro o significado desta situação, comparamos, em ambos os Grupos, as condições de finalização com a relação numérica relativa na perda da posse da bola (Quadro 4). No Grupo Sénior, verificamos uma diferença positiva quanto à intenção táctica - superioridade numérica relativa - porque há um aumento na sua incidência na acção final (verificada nos quadros anteriores), sendo as diferenças estatisticamente significativas entre os dois grupos. Permite confirmar a importância da competência individual e traduz a relevância de conduta adequada por parte do jogador responsável pela perda de posse da bola. Verifica-se que o Grupo Juvenil apresenta uma clara impossibilidade em transformar as condições de finalização em inferioridade numérica relativa.

Quadro 4 - Análise comparada das condições da perda de finalização e de perda da posse de bola que a equipa criou e de que tirou proveito em ataque posicional, com os valores de $\mathrm{F}$ e $\mathrm{p}$ referentes à comparação dos jogos.

\begin{tabular}{l|l|c|c|c|c}
\multicolumn{2}{|c|}{ Ataque posicional } & \multicolumn{2}{c|}{ Sénior } & \multicolumn{2}{c}{ Juvenil } \\
\hline & & $F$ & $p$ & $F$ & $p$ \\
\hline & superioridade numérica relativa & 16,010 & 0,000 & 0,914 & 0,352 \\
\hline & igualdade numérica relativa & 7,333 & 0,100 & 11,25 & 0,159 \\
\hline & inferioridade numérica relativa & 0,709 & 0,405 & 0,000 & 1,000
\end{tabular}

Relativamente às condições de finalização e perda da posse de bola em simetria absoluta no ataque posicional, temos uma configuração idêntica no método de jogo em ataque posicional, com a particularidade de serem dominantes as situações de igualdade numérica relativa, como condição de finalização para os dois Grupos e tal não se verificar no momento de perda da posse de bola.

\section{Ataque rápido}

Ao compararmos, em ambos os grupos, as condições de finalização com a relação numérica relativa na perda da posse da bola, registamos a única diferença estatisticamente significativa na igualdade numérica relativa para o Grupo Sénior $(0,024)$ (Quadro 5). Isto confirma que a conduta do jogador deste grupo permite tirar proveito das condições de relação numérica, convertendo as situações de igualdade numérica relativa em situações de superioridade numérica relativa.

Quadro 5 - Análise comparativa das condições de finalização e de perda da posse de bola que a equipa criou e de que tirou proveito em ataque rápido, com os valores de $\mathrm{F}$ e $\mathrm{p}$ referentes à comparação dos jogos.

\begin{tabular}{l|l|c|c|c|c}
\multicolumn{2}{l|}{ Ataque rápido } & \multicolumn{2}{|c|}{ Sénior } & \multicolumn{2}{|c}{ Juvenil } \\
\hline & Superioridade numérica relativa & 2,111 & 0,154 & 0,000 & 1,000 \\
\hline & igualdade numérica relativa & 5,516 & 0,024 & 0,062 & 0,806 \\
\hline & inferioridade numérica relativa & 0,06 & 0,809 & 0,072 & 0,791
\end{tabular}

\section{Contra-ataque}

No contra-ataque verificam-se grandes diferenças quanto à utilização deste método de jogo, pelos dois grupos. O valor da diferença encontrada em ambos os grupos, relativamente à relação numérica nas condições de finalização e perda da posse da bola, é estatisticamente significativo (Quadro 6).

Quadro 6 - Valores da análise das condições de finalização que a equipa criou em contra-ataque, com média (m) desvio-padrão (DP), amplitude, para cada um dos grupos, e os valores de $F$ e $p$ referentes à comparação dos grupos.

\begin{tabular}{|c|c|c|c|c|c|c|c|c|c|}
\hline \multicolumn{2}{|c|}{ Condições de finalização } & \multicolumn{3}{|c|}{ Seniores } & \multicolumn{3}{|c|}{ Juvenis } & \multirow[b]{2}{*}{$\mathrm{F}$} & \multirow[b]{2}{*}{$\mathrm{p}$} \\
\hline Contra Ataque & & $\mathrm{m}$ & $+\mathrm{DP}$ & amplitude & $\mathrm{m}$ & $+\mathrm{DP}$ & amplitude & & \\
\hline & Superioridade numérica relativa & 4,40 & $\pm 2,26$ & $1-9$ & 1,40 & $\pm 1,78$ & $0-6$ & 13,419 & 0,001 \\
\hline & Igualdade numérica relativa & 3,50 & $+1,93$ & $0-7$ & 0,70 & $+1,06$ & $0-3$ & 18,045 & 0,000 \\
\hline & Inferioridade numérica relativa & 1,05 & $\pm 1,10$ & $0-4$ & 0,80 & $+1,32$ & $0-4$ & 0,303 & 0,587 \\
\hline
\end{tabular}


Existe uma clara predisposição do Grupo Sénior para apresentar condições de finalização em superioridade numérica relativa (média de 4,40), embora as situações de igualdade numérica relativa apresentem um valor médio próximo (média de 3,50). A pouca expressão das situações de inferioridade numérica relativa deve estar associada à avaliação das condições para a realização do contra-ataque, efectuada num diminuto espaço de tempo para não inviabilizar a utilização deste método. Questões de competência, como temos referido, podem ajudar a explicar a razão do equilíbrio no sentido da criação da superioridade numérica relativa para o Grupo Juvenil. Assim temos resultados estaticamente significativos que realçam a preocupação do Grupo Juvenil em evitar as situações de igualdade relativa e recusar as de inferioridade numérica relativa, porque o tempo de decisão diminuto que a utilização deste método implica, só lhes permite a sua utilização quando a avaliação é inequívoca. O papel do guarda-redes no contra-ataque é determinante na criação de condições de finalização, porque o seu sucesso depende da velocidade com que a bola é reintroduzida no jogo (13), sendo mesmo esta a função principal do guarda-redes no jogo de ataque. As diferenças estatisticamente significativas, que aparecem no Quadro 7, relativas à perda da posse de bola, reportam-se às situações de superioridade e igualdade numérica relativa, tal como já se havia verificado quanto às condições de finalização, e confirmam a capacidade dos jogadores do Grupo Sénior em explorar o grande espaço, para tirar proveito ou criar superioridade numérica relativa.

Quadro 7 - Valores da análise da perda da posse de bola que a equipa criou em contra-ataque, com média (m) desvio-padrão (DP], amplitude, para cada um dos grupos, e os valores de $F$ e $p$ referentes à comparação dos grupos.

\begin{tabular}{l|l|c|c|c|c|c|c|c|c} 
Perda posse de bola & \multicolumn{4}{c|}{ Seniores } & \multicolumn{4}{c|}{ Juvenis } & \\
\hline Contra Ataque & & $\mathrm{m}$ & $+\mathrm{DP}$ & amplitude & $\mathrm{m}$ & $+\mathrm{DP}$ & amplitude & $\mathrm{F}$ & $\mathrm{p}$ \\
\hline & Superioridade numérica relativa & 6,30 & $\pm 2,39$ & $3-12$ & 1,60 & $\pm 1,96$ & $0-6$ & 28,916 & 0,000 \\
\hline & Igualdade numérica relativa & 2,00 & $\pm 1,56$ & $0-5$ & 0,90 & $\pm 1,29$ & $0-4$ & 3,709 & 0,064 \\
\hline & Inferioridade numérica relativa & 0,75 & $+1,02$ & $0-4$ & 0,50 & $+0,71$ & $0-2$ & 0,481 & 0,494
\end{tabular}

O quadro 8 , da análise comparada das condições de finalização e de perda da posse de bola, realça de forma estatisticamente significativa a capacidade do Grupo Sénior em jogar no grande espaço. O Grupo Sénior tira, claramente, proveito do grande espaço para alcançar a superioridade numérica relativa, com redução significativa das situações de igualdade numérica relativa e das situações de inferioridade numérica relativa, traduzida nas diferenças estatisticamente significativas entre a condição de finalização e a perda da posse de bola nas situações de superioridade $(0,014)$ e igualdade $(0,010)$ numérica relativa.

Quadro 8 - Análise comparada das condições de finalização e de perda da posse de bola que a equipa criou e de que tirou proveito em contra-ataque, com os valores de $F$ e p referentes à comparação.

\begin{tabular}{l|l|c|c|c|c}
\multicolumn{2}{l|}{ Contra ataque } & \multicolumn{2}{|c|}{ sénior } & \multicolumn{2}{c}{ juvenil } \\
\hline & & $F$ & $p$ & $F$ & $p$ \\
\hline & superioridade numérica relativa & 6,692 & 0,014 & 0,057 & 0,813 \\
\hline & igualdade numérica relativa & 7,308 & 0,010 & 0,144 & 0,709 \\
\hline & inferioridade numérica relativa & 0,345 & 0,560 & 0,403 & 0,534
\end{tabular}

A diferença, no que se refere à inferioridade numérica relativa, no Grupo Sénior não tem alteração significativa, até pela sua própria natureza, dado que as condições de finalização desfavoráveis deviam apontar para que a conduta do jogador se pautasse pela manutenção da posse de bola, mas questões de ordem diversa devem ter determinado a opção sem vantagem na relação numérica.

De facto, o ataque rápido e o contra-ataque realçam a dificuldade de interpretação do Grupo Juvenil dos métodos dependentes do jogo no grande espaço, porque das condições de finalização para a perda da posse de bola regista-se, em ambos os métodos, um aumento de situações de igualdade numérica relativa.

\section{As condições de finalização e a perda da posse de bola nas relações assimétricas absolutas. nos diferentes métodos de jogo}

Nos diferentes métodos de jogo, as relações de assimetria absoluta consideram a superioridade e a inferioridade numérica absoluta e na sua análise procu- 
ramos verificar a forma como os dois grupos se apresentaram: (i) perante o benefício da superioridade garantida à partida, em que é necessário mantê-la; e (ii) como lidam com o prejuízo, ou seja, a inferioridade numérica garantida à partida.

Ataque posicional em assimetria absoluta

Verificamos que as condições de finalização do ataque posicional, tanto em superioridade como em inferioridade numérica absoluta, permitem ao Grupo Sénior diferenciar-se do Grupo Juvenil, pela forma como lida com essa assimetria absoluta. O Grupo Sénior consolida a superioridade numérica absoluta de forma mais clara que o Grupo Juvenil e evita a inferioridade numérica relativa em situação de benefício, traduzindo-se esta última de forma estatisticamente significativa $(0,038)$. Em situação de prejuízo, o Grupo Sénior apresenta maior facilidade em lidar com essa mesma situação de inferioridade numérica relativa, parecendo evidente que apresenta capacidades para jogar em inferioridade, traduzindo-se também esta de forma estatisticamente significativa $(0,046)$.

As condutas de jogadores do Grupo Sénior permitem reduzir o prejuízo, com uma diferença estatisticamente significativa ao nível da perda de posse de bola em superioridade numérica relativa $(0,026)$, quando em situação de inferioridade numérica absoluta. Esta situação é distinta de as equipas estarem dotadas de um jogador especialista em rematar à baliza em condições de relação numérica desfavorável, já que nesta situação não se verifica a perda de posse de bola em inferioridade numérica relativa, mas sim em superioridade. Encontramos especialistas que rematam à baliza em inferioridade numérica relativa e outros que são capazes de converter uma situação de relação numérica desfavorável em favorável. Para ambos os grupos as diferenças entre as condições de finalização e de perda de posse de bola não apresentam diferenças estatisticamente significativas.

\section{A solicitação das diferentes linhas do sistema de jogo} A solicitação das diferentes linhas do sistema de jogo ofensivo, nos métodos de jogo em ataque posicional e ataque rápido, não regista grandes diferenças entre os dois grupos, na forma como as linhas são solicitadas. Verifica-se uma única diferença estatisticamente significativa $(0,014)$, relacionada com o ataque rápido em igualdade numérica relativa, na situação de perda de posse de bola. Esta é justificada porque a utilização da primeira linha em situações de ataque rápido pode ser importante para efectuar remate à baliza de zona idónea e pode ser suficiente para justificar esta situação.

\section{Número de passes de ataque anterior à perda da posse de bola em cada método de jogo}

O registo que efectuámos, do número de passes de ataque, pode constituir um indicador interessante se considerarmos que o número de passes de ataque é, de facto, reduzido (Quadro 9). Cerca 70\% dos passes não criam situações ameaçadoras, nem qualquer vantagem para as acções da equipa, e a finalização aparece suportada, associada a uma diversidade de número de passes relativamente restrita (12).

O Grupo Juvenil apresenta, no ataque posicional em igualdade numérica absoluta, menos passes, relativamente às mesmas condições para o Grupo Sénior. Grande parte das situações de finalização no Grupo Juvenil passa por um ou dois passes de ataque, enquanto que a incidência da solicitação no Grupo Sénior é de um a três passes, de forma muito equilibrada. O Andebol é cada vez mais alicerçado na aplicação de meios tácticos de grupo, os quais permitem múltiplas formas de conjugação de acções simples e que, diversificadas no seu encadeamento, tornam-se complexas quando suportadas por um forte e diversificado repertório técnico, proporcionando desta forma condições favoráveis à emergência da individualidade em processos mais criativos e espectaculares (42).
Ataque posicional \begin{tabular}{l|l|l|l|}
\hline Igualdade absoluta & Superioridade absoluta Inferioridade absoluta & Ataque rápido & Contra-ataque \\
\hline
\end{tabular}

\begin{tabular}{|c|c|c|c|c|c|c|c|c|c|c|}
\hline $\mathrm{n}^{\circ}$ passes & seniores & juvenis & seniores & juvenis & seniores & juvenis & seniores & juvenis & seniores & juvenis \\
\hline 0 & & $0.7 \%$ & & $3.5 \%$ & & & & & $2.6 \%$ & $6.1 \%$ \\
\hline 1 & $24,8 \%$ & & $18,6 \%$ & & $18,8 \%$ & & & $10,5 \%$ & $33,2 \%$ & \\
\hline 2 & $29,7 \%$ & & $40,0 \%$ & & $27,5 \%$ & & $4,9 \%$ & & $34,2 \%$ & \\
\hline 3 & $25,1 \%$ & $16,1 \%$ & $28,6 \%$ & & $27,5 \%$ & $6,7 \%$ & $4,9 \%$ & & $26,4 \%$ & $15,2 \%$ \\
\hline 4 & $10,0 \%$ & $8,4 \%$ & $8,6 \%$ & $3,5 \%$ & $11,6 \%$ & $13,3 \%$ & $34,2 \%$ & $15,8 \%$ & $3,7 \%$ & \\
\hline 5 & $5,7 \%$ & $2,5 \%$ & $4,3 \%$ & & $8,8 \%$ & & $48,8 \%$ & $10,5 \%$ & & \\
\hline 6 & $3,3 \%$ & $2,8 \%$ & & & $2,9 \%$ & & $2,5 \%$ & $5,3 \%$ & & \\
\hline 7 & $1,0 \%$ & $0,4 \%$ & & & $2,9 \%$ & & $4,9 \%$ & & & \\
\hline 8 & $0,5 \%$ & $0,4 \%$ & & & & & & & & \\
\hline
\end{tabular}

Quadro 9 - Percentagens da utilização do número de passes de ataque relativos à perda de posse de bola em cada método de jogo. 
A incidência de solicitação de passe de contra-ataque, idêntica para os dois grupos, parece ser uma vincada característica do método de jogo.

No ataque rápido surgem algumas dificuldades de interpretação se atendermos ao facto de o Grupo Juvenil apresentar uma grande variedade na utilização de recursos, traduzidos pelo número de passes. A transição para o terreno de ataque não é muito rápida, mas segura, pelo que só podemos considerar o número de passes para este efeito após a aceleração do ritmo que permitiu as condições de finalização.

\section{CONCLUSÕES}

Os resultados encontrados permitem concluir o seguinte:

1. Entre os dois grupos há divergência na utilização do grande espaço, verificando-se diferenças na exploração do contra-ataque, tendo este método algum relevo no jogo do Grupo Sénior. A quase totalidade do jogo de ataque no Grupo Juvenil verifica-se no pequeno espaço, sendo este método de jogo quase que exclusivo no jogo dos jovens.

2. A superioridade numérica relativa não é predominante como condição de finalização, para os dois grupos e nos diferentes métodos de jogo, mas é predominante na perda de posse de bola para o Grupo Sénior. Os jogadores do Grupo Sénior conseguem com maior frequência ultrapassar a oposição a partir das condições criadas pela equipa, sendo a competência individual que determina a predominância da superioridade numérica relativa na perda da posse de bola.

3. A utilização dos diferentes métodos de jogo em assimetria sofre alterações, com o quase desaparecimento do jogo no grande espaço, em ambos os grupos.

4. A primeira linha do sistema de jogo é mais importante no jogo de ambos os grupos, particularmente no método de jogo em ataque posicional, sendo manifestamente mais importante no jogo adulto.

5. O número de passes de ataque é reduzido, mas o Grupo Juvenil apresenta um menor número de passes de ataque, traduzindo um jogo com menor suporte na articulação das acções colectivas e de falta de continuidade no jogo de ataque.

\author{
CORRESPONDÊNCIA \\ Ireneu Moreira \\ Faculdade de Ciências do Desporto \\ e de Educação Física \\ Universidade do Porto \\ Gabinete de Andebol \\ Rua Dr. Plácido Costa, 91 \\ 4200.450 Porto, Portugal \\ imoreira@fcdef.up.pt
}




\section{BIBLIOGRAFIA}

1. Bana, P. (1996) Mondial masculin 1995: combinations tactiques. Approches du handball, 31: 2-7

2. Barbosa, J. (1999). A organização do jogo em andebol: estudo comparativo do processo ofensivo em equipas de alto nível, em função da relação numérica ataque-defesa. Dissertação de Mestrado. Faculdade de Ciências do Desporto e de Educação Física, Universidade do Porto (não publicado)

3. Barcenas, D. (1981). Táctica colectiva ofensiva. Barcelona: FEBM.

4. Bayer, C. (1983). Hanball. La formation du joueur. Paris: Vigot Editions

5. Bayer, C. (1990). Peut-on parler de specificité du handball feminin? Approches du Handball 5:18-20.

6. Castelo, J. (1994). Futebol - Modelo técnico-táctico do jogo. Lisboa: Edições FMH-UTL.

7. Castelo, J. (1996). Futebol, a organização do jogo. Edição do autor

8. Conceição, L. (1998) : Análise do Jogo de Andebol. Estudo comparativo do processo ofensivo em equipas de iniciadas e juvenis femininos. Dissertação de Mestrado. Faculdade de Ciências do Desporto e de Educação Física, Universidade do Porto (não publicado)

9. Costantini, D. (1995). Dossier spécial mundial - Le jeu de l' équipe de France. Approches du Handball, 27: 3-6.

10. Czerwinski, J. (1991). Structure du Handball. In IHF Trainers' and Chief-Referees' Symposium. Athènes: Éditions I. H. F, 55-62.

11. Czerwinski, J. (1993). El Balonmano. Técnica, Táctica y Entrenamiento. Barcelona: Paidotribo.

12. Czerwinski, J. (1994a). An analysis of the level of Technique and Tactics at the Woman's World Handball Championship in Oslo. Handball Periodical for Coaches, Referees and Lecteurs, 1: 4-11.

13. Czerwinski, J. (1994b). An analysis of the European Men's Championship - Portugal, June 1994: Technique and Tactics. Handball Periodical for Coaches, Referees and Lecteurs, 2: 7-16.

14. Czerwinski, J. (1995). Technical-Tactical analysis of Men's European Champions - Germany, September 1994. Handball Periodical for Coaches, Referees and Lecteurs, 2: 7-16.

15. Czerwinski, J. (1998). Statistical analysis of men's European Championship held in Italy 1998. Handball - EHF Periodical for Coaches, Referees and Lecteurs, 2: 10-18.

16. Czerwinski, J. (2000). Statistical analysis and remarks on the game character based on the European Championship in Croatia. Handball - EHF Periodical for Coaches, Referees and Lecteurs, 1: 5-10.

17. D’Hinaut, L. (1997). Conceitos e métodos da estatística. Vol.I. $2^{a}$ Edição. Lisboa: Fundação Calouste Gulbenkian.

18. F. A. P. (2001). Regras de Jogo. Lisboa: Federação de Andebol de Portugal.

19. Ferreira, J.; Queirós, C. (1982). Da formação à alta competição. Futebol em Revista. 3 (10): 35-43.

20. Fonseca, O. (1999). Andebol Português versus Andebol Mundial. Dissertação de Mestrado. Faculdade de Ciências do Desporto e de Educação Física, Universidade do Porto (não publicado).

21. García, J. L. A. (1998). Balonmano. Táctica grupal ofensiva. Concepto, estrutura y metodologia. Madrid: Gymnos.

22. García, J. L. A. (1999). La teoria escalonada de Roth: una vía imprescindible en el aprendizaje táctico del balonmano. In J. L. A. García, L. C. Ríos (Eds.) Jornadas de formación en balonmano, alternativas y factores a valorar el aprendizage (livro de resumos). Granada: FCAFD, Universidade de Granada, 159-177.
23. Garganta, J. (1997) Modelação táctica do jogo de Futebol. Estudo da organização da fase ofensiva em equipas de alto rendimento. Tese de Doutoramento. Faculdade de Ciências do Desporto e de Educação Física, Universidade do Porto (não publicado)

24. Germain, J-M (1997) Évolution du haut niveau féminin. Mondial 95. Approches du Handball, 37:15-29.

25. Germain, J-M (1997) Évolution du haut niveau féminin. Mondial 95. Approches du Handball, 38:15-34.

26. Germanescu, I. K. (s.d). Traité de Base pour Entraîneurs. Ecole Internationale de Handball de Fribourg. Fribourg: EIHF.

27. Krumbholz, O. (1995) Mondial espoir feminin. Approches du handball, 30: 33-36.

28. Landure, P; Curelli, J. (1996) Championnat d'Europe Masculin 1996. Approches du handball, 33:17-24/29-36.

29. Lassierra, G.; Ponz, J.M.; De Andrés, F. (1992). 1013 ejercicios y juegos aplicados al balonmano. Volumen I - Fundamentos y ejercicios individuales. Barcelona: Paidotribo.

30. Leitão, A. (1998). O Processo ofensivo no Andebol. Dissertação de Mestrado. Faculdade de Ciências do Desporto e de Educação Física, Universidade do Porto (não publicado).

31. Mortágua, L. (1999). Modelo de jogo ofensivo em Andebol. Estudo da organização das fases ofensiva em equipas seniores masculinas de alto rendimento portuguesas. Dissertação de Mestrado. Faculdade de Ciências do Desporto e de Educação Física, Universidade do Porto (não publicado).

32. Noteboom, T. (1995). Handball. Technique, pédagogie, entrâेnement. Paris : Amphora.

33. Oliveira, J. (1993). A análise do jogo de basquetebol. In J. Bento, A. Marques (Eds). A ciência do desporto, a cultura e o homem. Porto: Faculdade de Ciências do Desporto e de Educação Física, Universidade do Porto, 297-306.

34. Peñas, C.; Graña, P. (2000). O treino da velocidade no jogo de Andebol. http://www.efdeportes.com. Buenos Aires. 5, 28.

35. Queirós, C. (1983). Para uma teoria do ensino/treino do Futebol. Futebol em Revista, 4(1): 47-49.

36. Sanchez, F. (1991). Conteúdo do jogo. In Balonmano. Barcelona: COE, 30-161.

37. Seco, R. (1999). World Championship Analysis - Egypt 1999. EHF Periodical for Coaches, Referees and Lecteurs, 2: 2-7.

38. Silva, A. (1993). Caracterização do jogo ofensivo no andebol. Um estudo com atletas do escalão de formação. Tese de Doutoramento. Faculdade de Ciências do Desporto e de Educação Física, Universidade do Porto (não publicado).

39. Silva, M.P. (1999) A concepção de jogo. Tarefa fundamental do treinador na preparação da equipa. In F. Tavares (Ed.) Estudos 2. Porto: CEJD, Faculdade de Ciências do Desporto e de Educação Física, Universidade do Porto, 99-102.

40. Soares, S. (2001). Caracterização do processo ofensivo em equipas Nacionais e Internacionais. Monografia de licenciatura. Faculdade de Ciências do Desporto e de Educação Física, Universidade do Porto (não publicado).

41. Teodurescu, L. (1984). Problemas da teoria e metodologia nos jogos desportivos. Lisboa: Livros Horizonte.

42. Trosse, H. (1993). Balonmano. Entrenamiento, técnica e táctica. Barcelona: Martinez Roca.

43. Van der Mars (1989) Observer Reliability: Issues and Procedures. In: P. Darst, D. Zakrajsek, V. Mancini (eds). Analysis in Physical Education and Sport Instruction. Champaign: Human Kinetics, 53-79. 